\title{
Utilizing Photos As Program Themes
}

\author{
Kai-Yin Cheng* Ko-Yuan Chou* Sheng-Jie Luo* Bing-Yu Chen ${ }^{\dagger}$
}

National Taiwan University
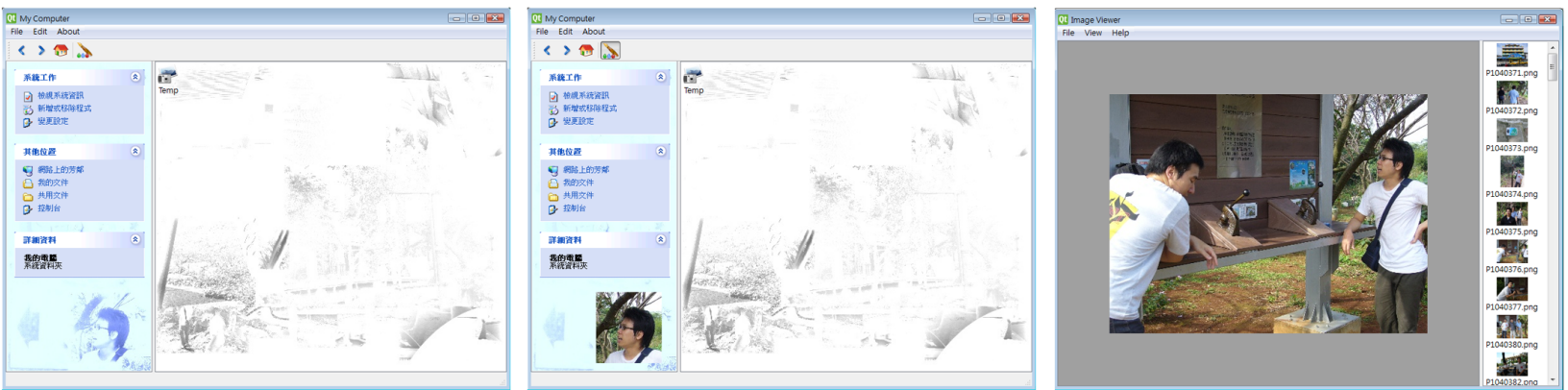

Figure 1: Left: inactive status. Middle: mouse hover one ROI under active status. Right: click one selected ROI to view the whole picture.

\section{Introduction}

Due to the development in digital technologies, people now can easily retain their valuable memory by taking pictures through digital cameras. The cheap digital storage also encourages people to take lots of photos as they want. However, due to the tremendous amount of digital photos, it is not easy for people to browse all of them. Therefore, some techniques are proposed to help people to enjoy the photos, although it may be difficult for some people to arrange a time slot to watch them intentionally. Hence, in this extended abstract, we propose a system, which can utilize the large number of photos as the program themes (background), so that people will not notice the synthesized background while they are working, but the program themes may still be able to remind their good memory when they taking a short rest.

\section{Design Principles}

In order to achieve this goal, some design principles are needed to be taken into account: 1) The synthesized background should not be salient and annoying that distracts people's attention while they are working. 2) The synthesized background should not interrupt users' work actively. 3) The synthesized background should obey the minimal aesthetics at least.

\section{System Overview and Implementation}

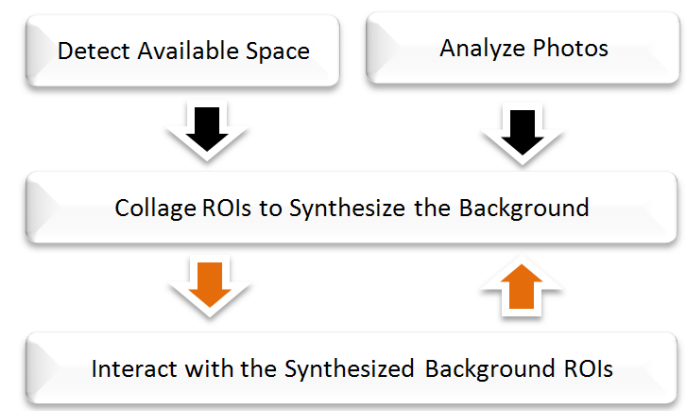

Figure 2: The overview of the system architecture.

As shown in Figure 2, our system first analyzes the photos to extract the ROIs [Ma and Zhang 2003]. Each found ROI is treated

*e-mail:\{keynes,koyuan,forestking $\} @$ cmlab.csie.ntu.edu.tw

†e-mail:robin@ntu.edu.tw as a basic element and has the chance to be collaged [Rother et al. 2006] with other ROIs to form a synthesized background image. The system then detects the program layout to determine the size of the background image to be generated. After synthesizing the ROIs as the background image, the system detects what areas in the program layout are occupied by active elements, such as icons or panels. In order to follow the design principle (1), the synthesized background should not cause any interference, the background areas with active elements should have less texture and less luminance. To follow the design principle (3), the system will change the synthesized background image's tone according to the color of the program's theme. The synthesized background image is static by default in order to follow the design principle (2) that the synthesized background should not interrupt users' work actively. Hence, if a user notices the background intentionally and wants to browse the image, he or she needs to activate the synthesized background image by clicking a functional button on the toolbar.

Figure 1 shows the simulation result. The most left picture is one result of the synthesized program background. After activating the background image, the user can use the mouse cursor to hover the ROI images as shown in the middle picture. If the user selects one ROI image, the picture viewer will be popped to let him or her browse the original picture which contains the selected ROI image.

\section{Conclusion and Future Work}

Users are embraced by their valuable memory while they are working through the synthesized background images generated by our proposed system, which transforms the tremendous troublesome photos into program background themes. In the future, we hope to improve the usability of the interaction model and consider more aesthetics issues.

\section{References}

MA, Y.-F., AND ZHANG, H.-J. 2003. Contrast-based image attention analysis by using fuzzy growing. In ACM Multimedia 2003 Conference Proceedings, 374-381.

Rother, C., Bordeaux, L., Hamadi, Y., and Blake, A. 2006. Autocollage. ACM Transactions on Graphics 25, 3, 847852. (SIGGRAPH 2006 Conference Proceedings). 